\title{
Eye Irritation Potential of Microglycine and Microglycine-Containing Ointments: An in vitro Study on Reconstructed Human Corneal Epithelium
}

This article was published in the following Dove Press journal:

Clinical Ophthalmology

\author{
Laura Ceriotti ${ }^{1}$ \\ Silvia Balzaretti \\ Salvatore Barone ${ }^{2}$ \\ Marisa Meloni (DI \\ 'VitroScreen, Milan, Italy; ${ }^{2}$ NTC S.r.l., \\ Milan, Italy
}

Correspondence: Laura Ceriotti VitroScreen, Via Mosè Bianchi 103, Milan 20149 , Italy

Tel +390289077608

Email laura.ceriotti@vitroscreen.com
Objective: To assess the eye tolerability of a buffered ophthalmic solution containing microglycine (sodium hydroxymethylglycinate, mwater $^{\mathrm{TM}}$ ) in an in vitro model.

Materials and Methods: A multiple endpoint analysis (MEA) approach was applied to the reconstructed human corneal epithelium (HCE) model. Sodium hydroxymethylglycinate solution $(0.04 \%)$ and two ophthalmic ointments containing microglycine (Protectorial, containing $0.02 \%$ of sodium hydroxymethylglycinate, and Edenight, containing $0.04 \%$ of sodium hydroxymethylglycinate) were investigated. The buffered solution and the ointments were tested on HCE after acute (one application in $24 \mathrm{hrs}$, followed or not by $16 \mathrm{hrs}$ of recovery) or repeated (one application per day for three consecutive days) exposures; benzalkonium chloride (BAK) $0.01 \%$ and saline isotonic solution were used as positive and negative controls, respectively. Cellular viability, trans-epithelial electrical resistance (TEER), lactate dehydrogenase (LDH) release and histo-morphology were evaluated.

Results: BAK $0.01 \%$ toxicity in HCE was confirmed for the $24+16 \mathrm{hrs}$ acute and repeated exposure protocols, while, after 24-hours acute treatment, only modifications of the superficial cell layer were visible compared with the negative control. Sodium hydroxymethylglycinate had a very good tolerability profile and a neutral impact on the corneal surface after acute or repeated exposure. The Protectorial and Edenight ointments preserved cell viability in the different exposure protocols, suggesting a good local tolerability profile. Modifications of the superficial layers were observed on histo-morphological analysis and confirmed by increased release of LDH after $24+16 \mathrm{hrs}$ acute exposure $(+65 \%$ and $+76 \%$ for Protectorial and Edenight, respectively) and TEER values after $24+16 \mathrm{hrs}$ and $72 \mathrm{hrs}$ exposure protocols. These results were dependent on the ointments' accumulation on the corneal epithelium due to their physical form (semi-solid) and lipophilic properties.

Conclusion: Sodium hydroxymethylglycinate, alone or as part of eye ointments, was found to be non-toxic after acute or repeated exposure in the reconstructed HCE model.

Keywords: BAK, biocompatibility, delayed toxicity, HCE, microglycine

\section{Plain Language Summary}

In recent years, an effort to reduce the use of animal testing in ophthalmology has led to the development of alternative experimental models, such as 3D reconstructed human corneal epithelium (HCE).

Microglycine (sodium hydroxymethylglycinate, mwater $^{\mathrm{TM}}$ ) is used to preserve Protectorial and Edenight ophthalmic ointments and to keep them free of contaminants.

In this study, we evaluated the effects of sodium hydroxymethylglycinate, Protectorial, and Edenight, on the structure and function of HCE model. 
After acute or repeated exposures, sodium hydroxymethylglycinate did not cause any significant change in the structure or function of HCE tissues. As a result, sodium hydroxymethylglycinate was considered to be non-toxic.

Protectorial and Edenight did alter the structure of the outermost layers of HCE tissues, and Protectorial also decreased its barrier function, compared with an isotonic saline solution. However, we hypothesized that these changes were the result of the ointments adhering to the epithelial surface, and not of their toxicity.

\section{Introduction}

After the Second World War, the use of preservatives in eye medications became common. Preservatives exert important effects, including prevention of the microbial growth that can occur after opening a container of multidose eye medication, keeping the formulation free from contaminants, and improving the safety of the product. However, the majority of preservatives are associated with adverse effects, inducing toxicity to the ocular cells or hypersensitivity, ${ }^{1}$ and have the potential to cause clinically relevant chronic or irreversible damage and ocular hyper-reactivity when used for long periods. ${ }^{1-3}$

Microglycine (sodium hydroxymethylglycinate) is a small, pale-yellow, water-soluble compound, available commercially as an aqueous solution (mwater ${ }^{\mathrm{TM}}$ ) with buffering properties and known antimicrobial effect. ${ }^{4}$ It is a derivative of the amino acid glycine and has broad-spectrum antimicrobial activity against both Gram-negative and Gram-positive bacteria, yeasts and moulds. ${ }^{5-8}$ Direct membrane interactions of peptides and amino acid-based surfactants produce antibacterial effects because these compounds have a net positive charge, which increases their interactions with various bacterial targets such as anionic lipids. ${ }^{9}$ However, anionic amino acid-based surfactants are also capable of producing antibacterial effects through membrane-altering actions. ${ }^{6-8,10,11}$

Protectorial and Edenight are ointments formulated for eye disorders, and both contain sodium hydroxymethylglycinate. As these ointments contain compounds other than sodium hydroxymethylglycinate, are lipophilic in nature and adhere to the surface of ocular epithelium due to their stickiness, their toxicity profiles may differ from that of sodium hydroxymethylglycinate alone.

Based on this background, we tested the hypothesis concerning the potential cytotoxicity of microglycine, alone or added to these eye ointments, to obtain information that could be relevant in clinical use. Therefore, the current study investigated the eye irritation potential of sodium hydroxymethylglycinate $0.04 \%$, Protectorial, and Edenight, using the human corneal epithelium (HCE) model. A multiple endpoints analysis (MEA) approach that included measurements of cellular viability, modifications of the barrier function, and histo-morphological analysis, was used, so that tissue function and morphology were evaluated.

\section{Materials and Methods Test Items and Controls}

Sodium hydroxymethylglycinate (buffered ophthalmic solution at $\mathrm{pH}$ 6.7-7.3) $0.04 \%$, Protectorial (containing $0.02 \%$ of sodium hydroxymethylglycinate), and Edenight (containing $0.04 \%$ of sodium hydroxymethylglycinate at $0.04 \%$, ) were tested (Table 1 ). Both ointments were provided by NTC S.r.l. (Milan, Italy).

Saline isotonic solution (sodium chloride 0.9\%; Eurospital, Trieste, Italy) and benzalkonium chloride (BAK; Merck Life Technology, Milan, Italy) $0.01 \%$ were used as negative and positive control, respectively., ${ }^{2,12-15}$

\section{Test System}

$\mathrm{HCE} / \mathrm{S} / 5$ (Human Corneal Epithelium, Small, $0.5 \mathrm{~cm}^{2}$ ) was purchased from Episkin (Lyon, France). Reconstructed HCE tissue derived from transformed human corneal keratinocytes was grown on a $0.5 \mathrm{~cm}^{2}$ inert permeable polycarbonate filter in the vertical direction, and then cultivated for 5 days in a supplemented, chemically defined medium at the airliquid interface, in order to form a structured epithelium. Immediately after delivery, HCE tissues were removed from nutrient agar and transferred into 6-well plates containing the growth medium provided by the manufacturer under sterile airflow conditions. Before any experiments were

Table I List of the Test Products

\begin{tabular}{|c|c|c|}
\hline Product Name & $\begin{array}{l}\text { Preservative } \\
\text { System }\end{array}$ & Manufacturer \\
\hline $\begin{array}{l}\text { Microglycine } 0.04 \% \\
\left(\text { mwater }^{T M}\right)\end{array}$ & $\begin{array}{l}0.04 \% \text { sodium } \\
\text { hydroxymethylglycinate }\end{array}$ & NTC S.r.l. \\
\hline $\begin{array}{l}\text { Protectorial isotonic eye } \\
\text { ointment with hyaluronic } \\
\text { acid } 0.4 \% \text { NTC }\end{array}$ & $\begin{array}{l}0.02 \% \text { sodium } \\
\text { hydroxymethylglycinate }\end{array}$ & NTC S.r.l. \\
\hline $\begin{array}{l}\text { Edenight hypertonic eye } \\
\text { ointment with hyaluronic } \\
\text { acid } 0.4 \% \text { NTC }\end{array}$ & $\begin{array}{l}0.04 \% \text { sodium } \\
\text { hydroxymethylglycinate }\end{array}$ & NTC S.r.l. \\
\hline
\end{tabular}


conducted, HCE tissues were equilibrated in an incubator at $37^{\circ} \mathrm{C}$ and $5 \% \mathrm{CO}_{2}$.

\section{Exposure Procedures}

To mimic realistic use conditions, the following exposure protocols have been included in the experimental design: i) acute, such as a single administration, followed or not by a recovery period; and ii) repeated, such as once per day application for three consecutive days.

For acute exposure, $30 \mu \mathrm{L}$ of each tested compound, namely saline solution, BAK $0.01 \%$, sodium hydroxymethylglycinate and ophthalmic ointments, were applied to the apical surface of HCE tissues. After $24 \mathrm{hrs}$ in the incubator at $37^{\circ} \mathrm{C}$ with $5 \% \mathrm{CO}_{2}$, the product was removed by washing with saline solution. Since this exposure emulated $24 \mathrm{hr}$ treatment, this is referred to as the $24 \mathrm{hr}$ protocol. The other acute exposure protocol involved $24 \mathrm{hr}$ exposure, followed by washing of product and incubation at $37^{\circ} \mathrm{C}$ with $5 \% \mathrm{CO}_{2}$ for $16 \mathrm{hrs}$. This is referred to as the $24+16 \mathrm{hr}$ protocol. The incubation period was selected as it was considered more useful for assessing the capacity of the corneal tissue to recover from toxic damage, and thus, for identifying reversible damage. ${ }^{16}$

For repeated exposure, because of the lipophilic nature of the ointments tested (containing $86 \%$ of lipophilic bases), the testing protocol was adapted for the semisolid formulations (which persist longer on the tissue surface), and the products were applied once per day and not twice per day as previously reported. ${ }^{13,16,17}$ Indeed, $30 \mu \mathrm{L}$ of saline solution, BAK $0.01 \%$ and active products were applied once every $24 \mathrm{hrs}$ to the apical surface of HCE tissues for $72 \mathrm{hrs}$ to mimic daily administration. This is referred to as the $72 \mathrm{hr}$ protocol. Before each application, the previous amount of residual product was removed by washing with saline solution.

\section{Cell Viability Quantification}

The 3-(4, 5-dimethylthiazolyl-2)-2, 5-diphenyltetrazolium bromide (MTT) assay was used to determine cell viability. This colorimetric assay measures the activity of succinate dehydrogenase, an enzyme expressed in the mitochondria of living cells. A modified version of the procedure originally described by Pauly and colleagues was used. ${ }^{12}$ In brief, HCE tissues were transferred to 24-well plates containing $300 \mu \mathrm{L}$ of $0.5 \mathrm{~g} / \mathrm{mL}$ MTT solution; $300 \mu \mathrm{L}$ of the same solution was also applied to the apical surface of HCE. Reconstituted tissues were incubated for $3 \mathrm{hrs}$ at $37^{\circ} \mathrm{C}$, and then transferred to 24 -well plates containing $750 \mu \mathrm{L}$ of isopropanol and $750 \mu \mathrm{L}$ isopropanol was added to the apical surface of HCE. After piercing the tissues with a tip, the plates were agitated for $2 \mathrm{hrs}$ in dark conditions at room temperature. The solutions were resuspended, and $200 \mu \mathrm{L}$ of extract transferred into a 96-well plate before the optical density (OD) at $570 \mathrm{~nm}$ was determined using the plate reader INFINITE M200 TECAN (software I-control, ver. 1.8). In the same 96-well plate, $200 \mu \mathrm{L}$ isopropanol was loaded (8 wells) and used as blank: isopropanol OD was subtracted from each OD value recorded. Experiments were conducted on duplicate HCE tissues for each series, and results were expressed as mean OD \pm standard deviation (SD) and as the proportion of cell viability measured with the negative control. The correlation between cell viability and eye irritation potential is described in the Organisation for Economic Co-operation and Development (OECD) Test Guideline No. 492. ${ }^{18}$ This guideline describes RhCE-based test methods validated and accepted by regulatory authorities for classification purposes. These methods include the SkinEthic ${ }^{\mathrm{TM}}$ HCE Eye Irritation Test (EIT), which uses the same HCE model as in this work. According to the protocol for liquid substances of the SkinEthic ${ }^{\mathrm{TM}}$ HCE EIT, a viability of $60 \%$ is the cut-off value for identifying substances that do not require classification and labelling due to causing eye irritation or serious damage (viability $>60 \%$ corresponds to classification as "no category" according to the United Nations [UN] Globally Harmonized System of Classification and Labelling of Chemicals [GHS]).

\section{Histo-Morphological Analysis: Hematoxylin and Eosin (H\&E) Staining}

Following the treatment procedure, tissues were washed with a saline solution and fixed in $10 \%$ neutral buffered formalin. Samples were embedded into paraffin blocks and sections of $5 \mu \mathrm{m}$ were sliced. Slides were stained with H\&E according to internal procedures. Light microscopy was used to analyze histological samples $(20 \times$ and $40 \times)$. Overall morphology and any modifications were analyzed on at least three sections of the same tissue and compared with the negative control.

\section{Membrane Integrity: Lactate Dehydrogenase (LDH) Quantification}

Membrane integrity was evaluated by measuring the presence of LDH in the extracellular medium using a commercially available Cytotoxicity Detection Kit (LDH; Roche, Monza, Italy). $100 \mu \mathrm{L}$ of medium was collected and added to a 96-well 
culture plate, together with $100 \mu \mathrm{L}$ of fresh medium, which served as a blank (technical triplicate). $100 \mu \mathrm{L}$ of the reaction mixture (freshly prepared by adding dye solution to the catalyst) was added to the medium in the 96-well culture plate, and incubated for 20 mins at room temperature in the dark; $50 \mu \mathrm{L}$ of stop solution was added to a 96-well plate to block the reaction. After shaking the plate for 10 seconds, absorbance was measured at $492 \mathrm{~nm}$ using $690 \mathrm{~nm}$ as the reference wavelength on the plate reader INFINITE M200 TECAN (software I-control, ver. 1.8). The blank OD was subtracted from each OD value recorded.

A standard curve defined using different LDH concentrations was used for $\mathrm{LDH}$ quantification: 125.00 $\mathrm{mU} / \mathrm{mL}, 62.50 \mathrm{mU} / \mathrm{mL}, 31.25 \mathrm{mU} / \mathrm{mL}, 15.60 \mathrm{mU} / \mathrm{mL}$, $7.80 \mathrm{mU} / \mathrm{mL}, 3.91 \mathrm{mU} / \mathrm{mL}$ and $1.95 \mathrm{mU} / \mathrm{mL}$. The $\mathrm{LDH}$ concentration in the samples was calculated by interpolating the biological replicate $\mathrm{OD}$ in the concentrationOD curve of LDH standards. Experiments were conducted on three HCE tissues and results were expressed as mean $\pm \mathrm{SD}$.

\section{Barrier Function Test: Trans-Epithelial Electrical Resistance (TEER)}

TEER was measured using the Millicell Electrical Resistance System (ERS) instrument (Merck Life Science, Milan, Italy) (range $0-20 \mathrm{k} \Omega$ ) before application, after product removal and after a period of recovery. The sample was placed in a 6-well plate containing $5.0 \mathrm{~mL}$ of saline solution, then $0.5 \mathrm{~mL}$ of saline solution was directly applied. The electrodes of the MillicellERS instrument were placed in the two chambers, and the reading on the display was reported. As blank (background control), the TEER value of a well without tissue was used. Triplicate measurements for each sample were recorded and experiments were performed with three tissues. Results were expressed as $\Omega \times \mathrm{cm}^{2} \pm \mathrm{SD}$ and as $\%$ difference from the baseline TEER values for each test item and control series at $\mathrm{t}=0$, which was considered as $100 \%$.

\section{Statistical Analysis}

Statistical analysis was performed using Student's $t$-test. P-values $<0.01$ and $<0.05$ compared with the negative control are presented in the figures describing cell viability, LDH release and TEER measurements. Individual data for each assay are available upon request.

\section{Results}

\section{Cell Viability: MTT Assay}

Figure 1 shows the results of the MTT test conducted on HCE after acute or repeated exposures. Conventionally, the negative control (saline solution) was assigned $100 \%$ cell viability. BAK $0.01 \%$ reduced cellular viability to $70.4 \%$ compared with the negative control after 24 hrs. After 24 $+16 \mathrm{hr}$ recovery, the residual viability was $77.4 \%$; after 72 hrs, viability was $57.7 \%$. Concerning sodium hydroxymethylglycinate, cellular viability was not significantly reduced (below 60\%) following any of the three exposure protocols, ranging from $77 \%$ to $92 \%$. Similarly, across the three exposure protocols, Protectorial and Edenight ointments did not reduce cell viability below $60 \%$. Following the $24 \mathrm{hr}, 24+16 \mathrm{hr}$ and $72 \mathrm{hr}$ exposures to these ointments, residual viability was $83-93 \%, 87-97 \%$ and $75-77 \%$ compared with the negative control, respectively.

\section{Histo-Morphological Analysis: H\&E Staining}

Representative images of selected vertical sections of HCE tissues at different times are shown in Figure 2.

The morphology of the tissue and its thickness did not change after $24 \mathrm{hr}$ treatment with the negative control. The increased thickness observed after $72 \mathrm{hr}$ treatment was related to the physiological growth of the 3D culture. The histo-morphological analysis did not show significant modifications between the three exposure protocols, confirming the neutral action of the saline solution in acute and repeated treatments.

BAK $0.01 \%$ induced significant modifications and signs of toxicity in the superficial layer of the epithelial barrier, including the loss of cell-to-cell connections, modified cell structure and the presence of several pyknotic nuclei, after $24 \mathrm{hr}$ treatment and $24+16 \mathrm{hr}$ protocol. These toxic effects worsened and became visible after $72 \mathrm{hr}$ treatment.

No toxicity was observed with sodium hydroxymethylglycinate compared with BAK $0.01 \%$ in the corneal epithelium. Sodium hydroxymethylglycinate induced the formation of superficial pyknotic nuclei surrounded by mucins. No further modifications of the HCE tissue were observed either after acute treatments or after $72 \mathrm{hr}$ exposure.

Both ointments caused morphological modifications exclusively in the corneal epithelium surface, but the 


\section{MTT on HCE: acute and repeated exposures}

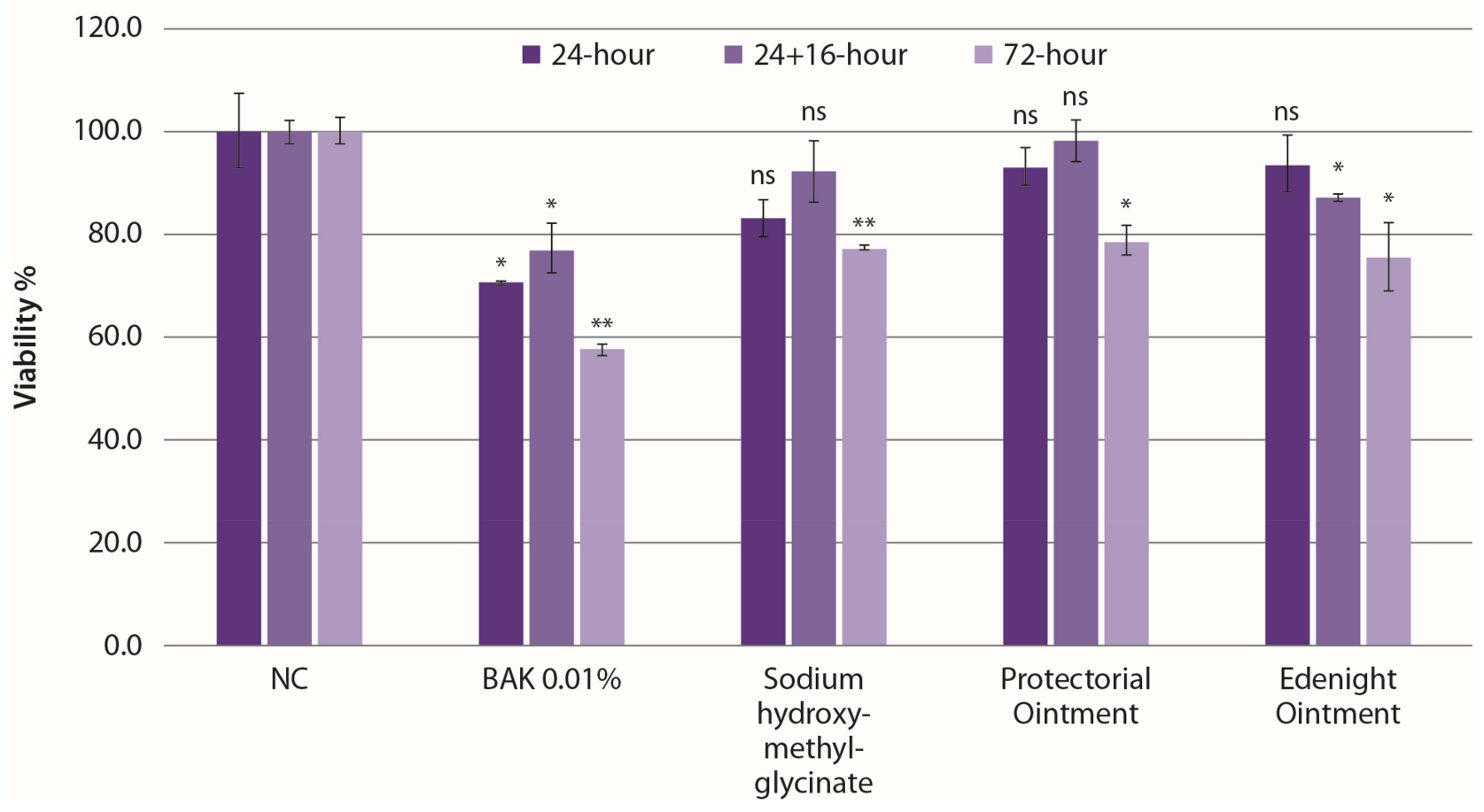

Figure I Residual cell viability (\%) after acute exposures ( $24 \mathrm{hr}$ treatment, $24+16 \mathrm{hr}$ recovery) and repeated exposure (72 hrs). Experiments were conducted on duplicate HCE tissues. Statistical analysis was performed using Student's $t$-test. ${ }^{*} \mathrm{p}<0.05$; ${ }^{*} \mathrm{p}<0.01$.

Abbreviations: HCE, human corneal epithelium; MTT, 3-(4, 5-dimethyl thiazolyl-2)-2, 5-diphenyltetrazolium bromide; NC, negative control; ns, not significant.

basal layer was preserved during and after all three exposure protocols investigated.

\section{Membrane Integrity: LDH Release Quantification}

A significant increase in LDH release was observed after $24+16 \mathrm{hr}$ exposure for all the products tested (including BAK) compared with the negative control $(+65 \%$ and $+76 \%$ for Protectorial and Edenight, respectively), while no significant changes were observed for the $24 \mathrm{hr}$ treatment, as reported in Table 2.

Figure 3 shows the relative amount of LDH released each day corresponding to culture medium change every $24 \mathrm{hrs}$. The numbers included in the graph represent the sum of the LDH amount collected at each time point.

The LDH release measured with the negative control was considered as the baseline LDH release level at each time point, namely $98.7 \mathrm{mU} / \mathrm{mL}, 87.1 \mathrm{mU} / \mathrm{mL}$ and 105.2 $\mathrm{mU} / \mathrm{mL}$ after $24 \mathrm{hrs}, 48 \mathrm{hrs}$ and $72 \mathrm{hrs}$, respectively, for a total of $291 \mathrm{mU} / \mathrm{mL}$.

BAK $0.01 \%$ induced a greater release of $\mathrm{LDH}$ in the extracellular medium compared with the negative control.
At 72 hrs, a two-fold increase was observed, which was consistent with the damage at the cellular membrane level, as reported in the histo-morphological analysis.

At $24 \mathrm{hrs}$ and $48 \mathrm{hrs}$, LDH release was not different from BAK $0.01 \%$ for all the products tested (ranging from $104 \mathrm{mU} / \mathrm{mL}$ to $119 \mathrm{mU} / \mathrm{mL}$ ). Similarly, compared with the negative control, no differences in LDH release were observed after $72 \mathrm{hr}$ treatment with sodium hydroxymethylglycinate $(111.2 \mathrm{mU} / \mathrm{mL})$, Protectorial $(104.6 \mathrm{mU} / \mathrm{mL})$ or Edenight (109.4 mU/mL).

\section{Barrier Integrity: TEER Measurement}

A baseline mean $\pm \mathrm{SD}$ value of $128 \pm 14 \Omega \times \mathrm{cm}^{2}$ was recorded for HCE tissues before treatment $(\mathrm{t}=0, \mathrm{~N}=45$ tissues tested). Mean TEER values for the negative control after $24 \mathrm{hr}, 24+16 \mathrm{hr}$ and $72 \mathrm{hr}$ treatment are reported in Table 3 as examples of TEER measurements under the experimental conditions adopted in this study. TEER values at the end of each exposure procedure, expressed as the difference from baseline values at $\mathrm{t}=0$ considered as $100 \%$, are reported in Figure 4.

BAK $0.01 \%$ exhibited TEER values similar to the negative control after $24 \mathrm{hr}$ treatment, but significantly $(\mathrm{p}<0.01)$ 


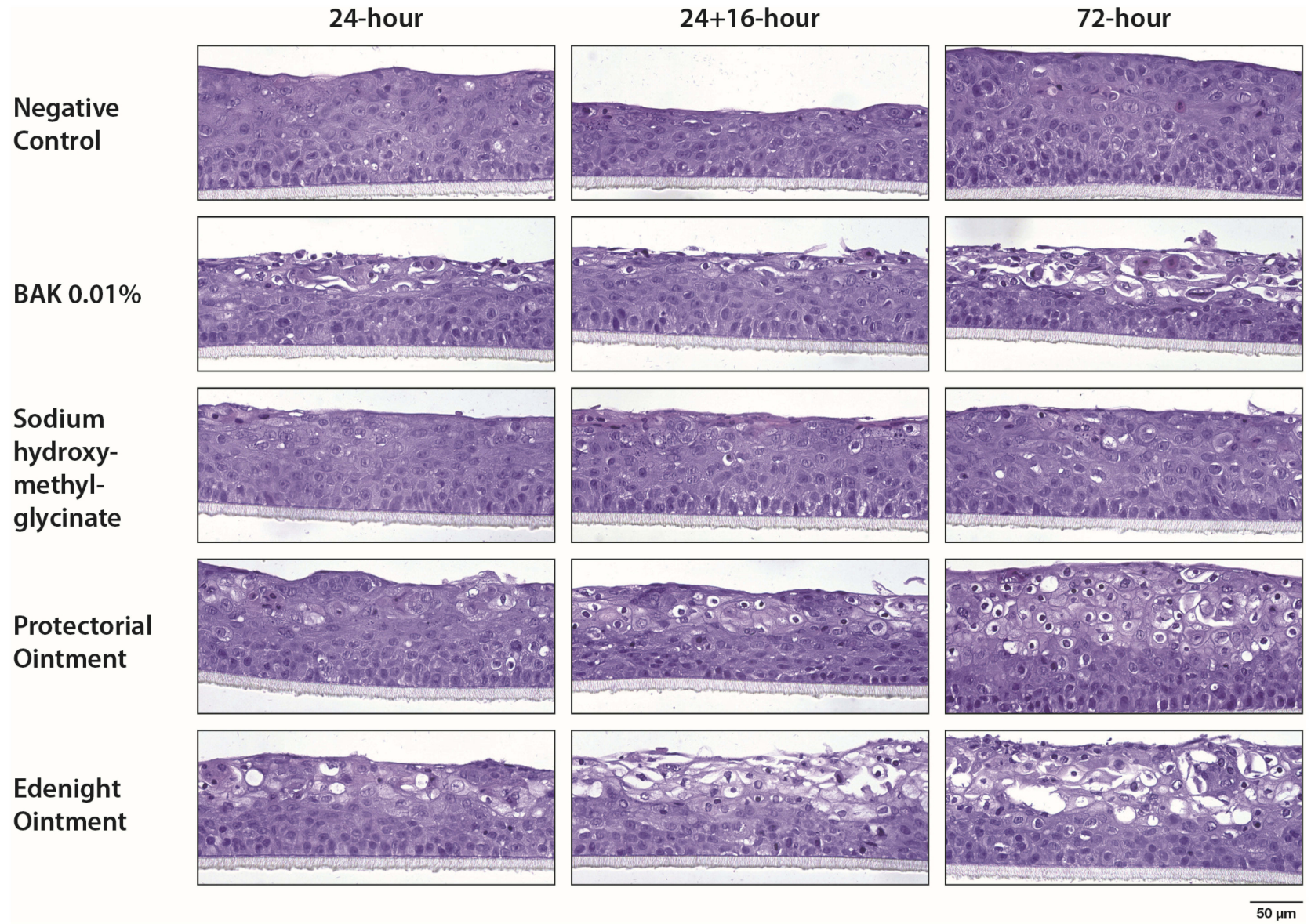

Figure 2 Selected images of HCE tissue sections after $24 \mathrm{hr}, 24+16 \mathrm{hr}$ and $72 \mathrm{hr}$ exposures (H\&E staining, 40x). Experiments were conducted on a single HCE tissue. Abbreviations: HCE, human corneal epithelium; H\&E, hematoxylin and eosin.

lower values compared with the negative control after 24 $+16 \mathrm{hr}$ protocol $(-71 \%)$ and after $72 \mathrm{hr}$ treatment $(-86 \%)$.

At $24 \mathrm{hrs,} \mathrm{TEER} \mathrm{values} \mathrm{for} \mathrm{sodium} \mathrm{hydroxymethylgly-}$ cinate, and Protectorial ointment were similar to the negative control, while the TEER value for Edenight ointment was significantly $(\mathrm{p}<0.05)$ different.

After repeated exposure (72 hrs), Protectorial ointment induced an increase in TEER that was greater than the negative control $(+45 \%)$, while TEER values for sodium hydroxymethylglycinate and Edenight ointment were in the same range as for the negative control.

\section{Discussion}

Historically, animal models have been used to assess the effects of chemicals on human health. However, the Draize in vivo eye irritation test in rabbits (OECD Test Guideline

Table 2 Lactate Dehydrogenase Release, Expressed in $\mathrm{mU} / \mathrm{mL}$ for the Acute Procedures: $24 \mathrm{hr}$ Treatment and 24+16 hr Recovery. Experiments Were Conducted on Triplicate Human Corneal Epithelium Tissues. Statistical Analysis Was Performed Using Student's $t$-Test

\begin{tabular}{|c|c|c|c|c|}
\hline & \multicolumn{4}{|c|}{ LDH Release (mU/mL) } \\
\hline & $24 \mathrm{hrs}$ & p-value & $24+16 \mathrm{hrs}$ & p-value \\
\hline Negative control (saline) & $93.8 \pm 5.6$ & & $60.3 \pm 4.0$ & \\
\hline BAK $0.01 \%$ & $110.9 \pm 4.1$ & $>0.05$ (ns) & $100.6 \pm 4.4$ & $<0.05$ \\
\hline Sodium hydroxymethylglycinate & $93.9 \pm 2.4$ & $>0.05$ (ns) & $99.8 \pm 0.8$ & $<0.01$ \\
\hline Protectorial ointment & $91.4 \pm 5.8$ & $>0.05$ (ns) & $98.8 \pm 0.7$ & $<0.01$ \\
\hline Edenight ointment & $97.3 \pm 1.7$ & $>0.05$ (ns) & $105.8 \pm 3.4$ & $<0.01$ \\
\hline
\end{tabular}

Abbreviations: BAK, benzalkonium chloride; LDH, lactate dehydrogenase; ns, not significant. 


\section{LDH release: repeated exposure within 72 hours}

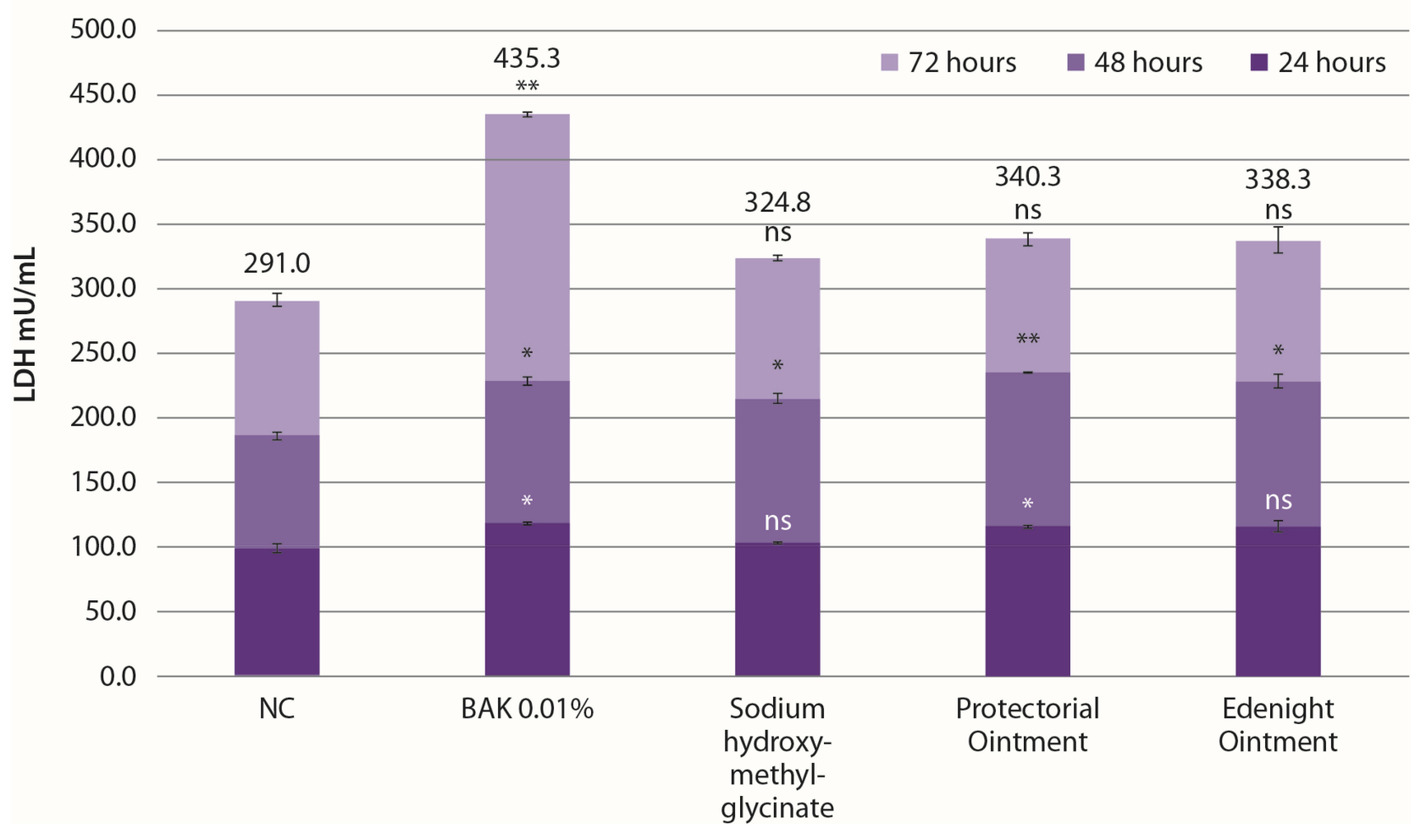

Figure 3 Cumulative LDH release quantification, expressed in $\mathrm{mU} / \mathrm{mL}$ for repeated exposure ( 24 hrs: Ist medium exchange; 48 hrs: 2 nd medium exchange; 72 hrs: 3 rd medium exchange). Experiments were conducted on triplicate HCE tissues. The number reported in the graphs represents the sum of LDH release amounts for each time point. Statistical analysis was performed using Student's $t$-test. ${ }^{*} p<0.05 ;{ }^{*} p<0.01$.

Abbreviations: HCE, human corneal epithelium; LDH, lactate dehydrogenase; NC, negative control; ns, not significant.

No. 405$)^{19}$ has been criticized, ${ }^{20-25}$ and multiple alternative methods for assessing eye irritation have been developed and validated in order to replace it or to reduce the number of animals used, according to the requirements of the Directive 2010/63/EU. ${ }^{26}$ These methods are now available for use as part of the Integrated Approach to Testing and Assessment (IATA) for classification and labelling purposes. ${ }^{27}$ Such validated methods include tests which use 3D reconstructed human corneal-like epithelia (RhCE) as biological models as described in the OECD Test Guideline No. $492,{ }^{18}$ demonstrating the relevance of these tissues for hazard identification. ${ }^{28,29}$

Table 3 TEER Mean Values for the Negative Control After $24 \mathrm{hr}$, $24+16 \mathrm{hr}$ and $72 \mathrm{hr}$ Treatment Expressed as $\mathbf{\Omega} \times \mathrm{cm}^{2}$. Experiments Were Conducted on Triplicate Human Corneal Epithelium Tissues

\begin{tabular}{|l|l|}
\hline & TEER Mean Values, $\mathbf{\Omega \times \mathbf { c m } ^ { 2 }}$ \\
\hline $24 \mathrm{hrs}$ & $176.50 \pm 9.20$ \\
$24+16 \mathrm{hrs}$ & $221.56 \pm 22.00$ \\
$72 \mathrm{hrs}$ & $198.00 \pm 13.34$ \\
\hline
\end{tabular}

Abbreviation: TEER, trans-epithelial electrical resistance.
The 3D human models can be used to identify ocular irritation due to their high level of morphological and biological similarity to the real human ocular epithelium. These human-origin models are more relevant and predictive in the assessment of eye irritation potential than monolayers of corneal epithelial cells or cells derived from other organs. RhCE models have been developed for use in permeability and safety studies during ophthalmic drug development. ${ }^{30}$

The MEA approach has been developed using HCE and acute and repeated exposure, to provide a predictive tool for assessing the short- and long-term eye irritation potential to the pharmaceutical industry. ${ }^{13}$ The in vitro identification of subclinical effects reduces the risks associated with the long-term use of ophthalmic products. ${ }^{12}$ Furthermore, the MEA approach relies on a mechanistic understanding of low or very low irritation potential to define tolerance. ${ }^{2,13,14,16,17}$

This study evaluated the eye irritation potential of microglycine, a new buffered ophthalmic solution with weak preservative properties, and of two ophthalmic ointments containing microglycine at different concentrations: $0.02 \%$ in the Protectorial and $0.04 \%$ in the Edenight ointment. The 


\section{TEER: acute and repeated exposures}

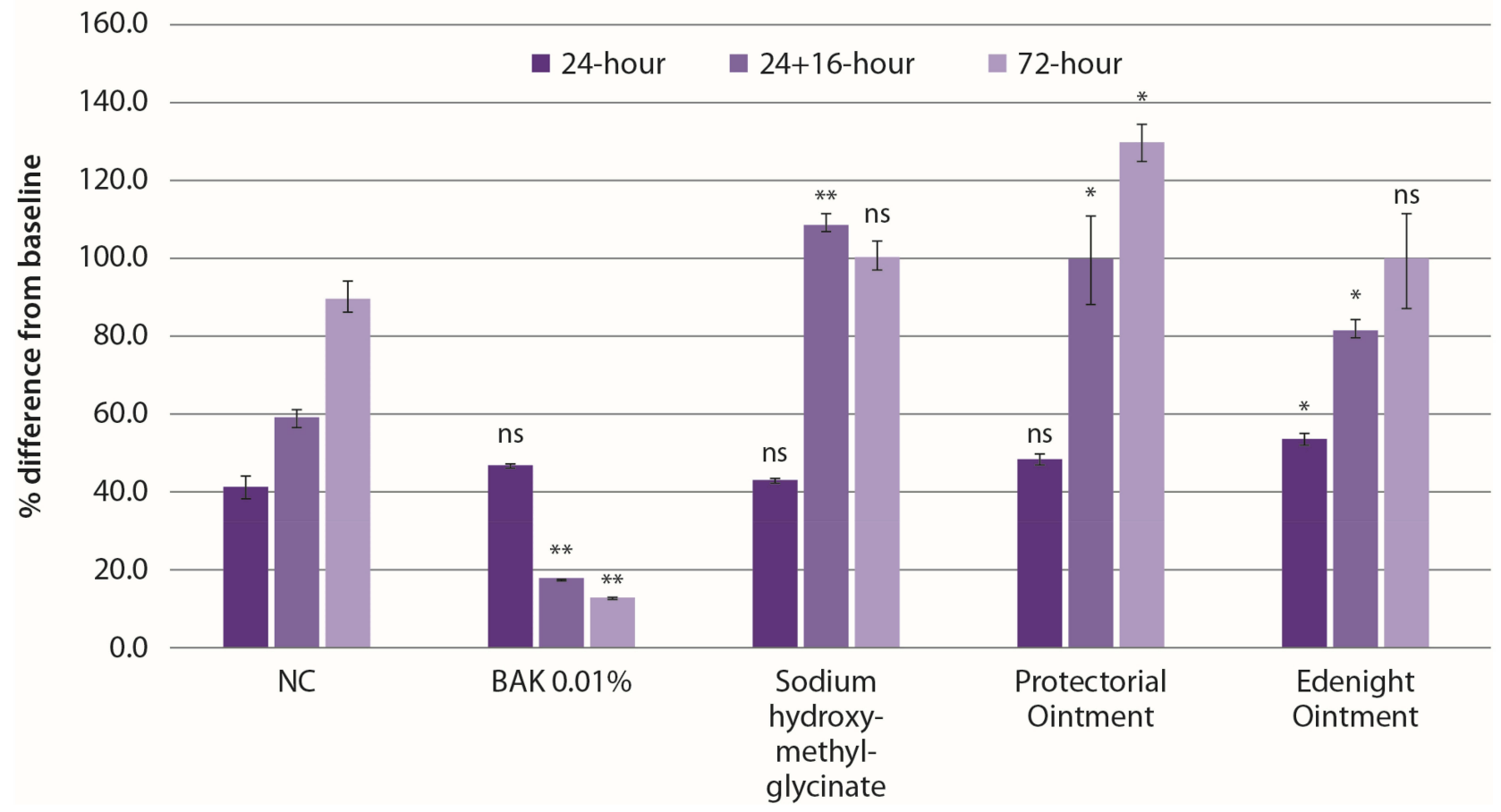

Figure 4 Difference in TEER from baseline ( $\mathrm{t}=0 \mathrm{hrs})$ for acute $(24 \mathrm{hr}$ treatment, $24+16 \mathrm{hr}$ recovery) and repeated $(72 \mathrm{hrs})$ exposures expressed in \%. Experiments were conducted on triplicate HCE tissues. Statistical analysis was performed using Student's $t$-test. ${ }^{*} p<0.05 ;{ }^{* *} p<0.01$.

Abbreviations: HCE, human corneal epithelium; NC, negative control; ns, not significant; TEER, trans-epithelial electrical resistance.

MEA approach, consisting of histo-morphological analysis, LDH release assessments and TEER measurements, was used to integrate the MTT results, providing more complete information about the overall tissue response at the ocular surface level than cell viability data alone.

The overall results of the MEA analysis confirmed the toxic effects of BAK $0.01 \%$ on the epithelial surface and its relevance as a positive control for evaluating ophthalmic products. $^{12,14}$ In our study, the unaffected cellular viability, tissue morphology and barrier permeability provide the evidence that sodium hydroxymethylglycinate has no local toxicity on the corneal surface after both acute and repeated exposures.

The MTT assay confirmed that both ophthalmic ointments containing microglycine did not induce significant cytotoxic damage. At $72 \mathrm{hrs}$, sodium hydroxymethylglycinate and the Protectorial and Edenight ointments had shown similar LDH release, which was found to be significantly lower than the LDH release observed with BAK $0.01 \%$, but higher than that observed with the negative control (approximately $+14 \%$ ). LDH measurement results were consistent with cellular membrane changes occurring in the whole thickness of HCE tissue, which did not depend on a toxic effect.
TEER outcomes reflect the overall resistance of the ocular barrier that results both from the integrity of tight junctions and from epithelial thickness. The lower TEER values reported for BAK $0.01 \%$ compared with the negative control after $24+16 \mathrm{hr}$ recovery $(-71 \%)$ and after $72 \mathrm{hr}$ treatment $(-86 \%)$ are consistent with the modification of the barrier properties after $24+16 \mathrm{hr}$ recovery and repeated treatment, as confirmed by histo-morphological analysis and $\mathrm{LDH}$ release.

The TEER increase registered for the Protectorial and Edenight ointments, after $24+16 \mathrm{hr}$ recovery and after $72 \mathrm{hr}$ treatment is related to: i) the physiological increase in epithelial thickness during culture, and ii) modification of epithelial morphology with water accumulation in the wing cell layer as visible in the H\&E images. On the other hand, increasing TEER values of the negative control at these time points depend exclusively on the physiological increase in epithelial thickness during culturing, while the epithelial structure remained intact.

The MEA approach applied to both the Protectorial and Edenight ointments demonstrated a good local tolerability profile after both acute and repeated exposures, consistent with preservative-free product features. However, the 
MEA evaluation reported that both ointments: i) induced morphological modifications exclusively in the corneal epithelium surface; ii) increased LDH release during 24 $+16 \mathrm{hr}$ recovery; and iii) increased TEER values more than the negative control during the $24+16 \mathrm{hr}$ recovery and the $72 \mathrm{hr}$ exposure protocol. These results were mainly due to the physical form (semi-solid) and chemical composition (presence of lipophilic ingredients in the ointments at $86 \%$ ) of the products, which resulted in strong adhesion to the corneal epithelium. This finding could be interpreted as an experimental bias. It is worth mentioning that after repeated exposure, it was not possible to completely remove all residue of the products that accumulated on the corneal surface, influencing tissue thickness.

Most ophthalmic preparations contain preservatives in order to prevent contamination and biodegradation, and to maintaining drug potency. ${ }^{31}$ There are four main classes of preservatives: detergents (BAK), oxidants (sodium perborate), chelating agents (methylparaben), and metabolic inhibitors (stabilized thiomersal). ${ }^{10,11} \mathrm{BAK}$ is by far the most common preservative used in topical ophthalmic preparations. $^{31}$

Few studies have compared the toxicity of preservatives used in ophthalmic preparations. The effects of BAK, methyl paraben, sodium perborate, chlorobutanol and stabilized thiomersal on immortalized human conjunctival and corneal epithelial cells were evaluated in a study conducted by Epstein and colleagues. ${ }^{31}$ When the most commonly used concentrations were considered, stabilized thiomersal was the most toxic preservative evaluated, followed by BAK, chlorbutanol, methyl paraben and sodium perborate. All preservatives caused some degree of tissue damage even at low concentrations. ${ }^{31}$

The MEA approach adopted in this study permitted the evaluation of tissue response to treatment after acute application, as well as of recovery after acute exposure and of potential cumulative effects associated with prolonged treatment. Furthermore, it allowed for an evaluation of cytotoxicity and irritation potential in one assay and compliance with the Directive $2010 / 63 / \mathrm{EU}^{26}$ promoting the use of non-animal testing methods, while also overcoming the well-known limitations of cell monolayers and in vivo approaches. $^{26}$

However, this study had some limitations, including the in vitro design, the lack of inflammation and biomarker assessment, except for LDH. Therefore, other studies should be designed and performed to respond to these unanswered questions.
On the other hand, this is one of the few studies that evaluated the tolerability of sodium hydroxylmethylglycinate when applied to human corneal epithelium cells.

\section{Conclusions}

The current in vitro study demonstrated that buffered sodium hydroxymethylglycinate solution was non-toxic in the HCE model after acute or repeated exposure. The ophthalmic formulations containing sodium hydroxymethylglycinate, such as the Protectorial and Edenight ointments, could also be considered non-toxic after acute or repeated treatments.

\section{Abbreviations}

BAK, Benzalkonium chloride; EIT, Eye irritation test; ERS, Electrical Resistance System; HA, Hyaluronic acid; HCE, Human corneal epithelium; H\&E, Hematoxylin and eosin; IATA, Integrated Approaches to Testing and Assessment; LDH, Lactate dehydrogenase; MEA, Multiple endpoint analysis; MTT, (3-(4, 5-dimethylthiazolyl-2)-2, 5-diphenyltetrazolium bromide); OECD, Organization for Economic Cooperation and Development; SD, Standard deviation; TEER, Trans-epithelial Electrical Resistance; TG, Test guideline; UN GHS, United Nations Globally Harmonized System of Classification and Labelling of Chemicals.

\section{Data Sharing Statement}

No additional data from this study are available.

\section{Acknowledgments}

Authors would like to thank Matthew Weitz, of Springer Healthcare Communications, for medical writing assistance. This assistance was funded by NTC S.r.l., Italy.

\section{Author Contributions}

Laura Ceriotti: Manuscript writing. Silvia Balzaretti: Experimental phase and data analysis. Salvatore Barone: Support for manuscript writing. Marisa Meloni: Conceptualization and data discussion. All authors contributed to data analysis, drafting or revising the article, gave final approval of the version to be published, and agreed to be accountable for all aspects of the work.

\section{Funding}

No specific funding was received for this study. The medical writing assistance was funded by NTC S.r.l., Italy. 


\section{Disclosure}

Laura Ceriotti, Silvia Balzaretti and Marisa Meloni are employees of VitroScreen, Milan, Italy. Salvatore Barone is an employee of NTC S.r.l., Italy. The authors report no other conflicts of interest in this work.

\section{References}

1. Velpandian T. Preservatives for topical ocular drug formulations. In: Velpandian T, editor. Pharmacology of Ocular Therapeutics. Adis; 2016:419-430

2. Baudouin C. Detrimental effect of preservatives in eyedrops: implications for the treatment of glaucoma. Acta Ophthalmol. 2008;86 (7):716-726. doi:10.1111/j.1755-3768.2008.01250.x

3. Rossi GC, Pasinetti GM, Scudeller L, Raimondi M, Lanteri S, Bianchi PE. Risk factors to develop ocular surface disease in treated glaucoma or ocular hypertension patients. Eur J Ophthalmol. 2013;23 (3):296-302. doi:10.5301/ejo.5000220

4. Rapuano PB, Scanameo AH, Amponin DE, et al. Antimicrobial studies using the therapeutic tissue cross-linking agent, sodium hydroxymethylglycinate: implication for treating infectious keratitis. Invest Ophthalmol Vis Sci. 2018;59(1):332-337. doi:10.1167/iovs.17-23111

5. Paulus W. Microbicides for the Protection of Materials: A Handbook. Netherlands: Springer; 1993.

6. Jenssen H, Hamill P, Hancock RE. Peptide antimicrobial agents. Clin Microbiol Rev. 2006;19(3):491-511. doi:10.1128/CMR.00056-05

7. Sanchez L, Mitjans M, Infante MR, Vinardell MP. Potential irritation of lysine derivative surfactants by hemolysis and $\mathrm{HaCaT}$ cell viability. Toxicol Lett. 2006;161(1):53-60. doi:10.1016/j.toxlet.2005.07.015

8. Sanchez L, Mitjans M, Infante MR, Garcia MT, Manresa MA, Vinardell MP. The biological properties of lysine-derived surfactants. Amino Acids. 2007;32(1):133-136. doi:10.1007/s00726006-0318-x

9. Noecker R. Effects of common ophthalmic preservatives on ocular health. Adv Ther. 2001;18(5):205-215. doi:10.1007/BF02853166

10. Spickett C. Studies of Cellular Responses to Purite and Other Preservatives. Strathclyde, UK: University of Strathclyde; 2001.

11. Asbell P Artificial tears: past, present, and future. 5th International Symposium on Ocular Pharmacology and Therapeutics (ISOPT); Monaco: Monte Carlo; 2004.

12. Pauly A, Meloni M, Brignole-Baudouin F, Warnet JM, Baudouin C. Multiple endpoint analysis of the 3D-reconstituted corneal epithelium after treatment with benzalkonium chloride: early detection of toxic damage. Invest Ophthalmol Vis Sci. 2009;50(4):1644-1652. doi:10.1167/iovs.08-2992

13. Meloni M, Pauly A, Servi BD, Varlet BL, Baudouin C. Occludin gene expression as an early in vitro sign for mild eye irritation assessment. Toxicol in Vitro. 2010;24(1):276-285. doi:10.1016/j.tiv.2009.08.016

14. Liang H, Pauly A, Riancho L, Baudouin C, Brignole-Baudouin F. Toxicological evaluation of preservative-containing and preservative-free topical prostaglandin analogues on a three-dimensionalreconstituted corneal epithelium system. Br J Ophthalmol. 2011;95 (6):869-875. doi:10.1136/bjo.2010.189449

15. Postnikoff CK, Pintwala R, Williams S, Wright AM, Hileeto D, Gorbet MB. Development of a curved, stratified, in vitro model to assess ocular biocompatibility. PLoS One. 2014;9(5):e96448. doi:10.1371/journal.pone.0096448

16. Meloni M, Balzaretti S, Ceriotti L. Medical devices biocompatibility assessment on HCE: evidences of delayed cytotoxicity of preserved compared to preservative free eye drops. Regul Toxicol Pharmacol. 2019;106:81-89. doi:10.1016/j.yrtph.2019.04.022
17. Meloni M, Cattaneo G, De Servi B. Corneal epithelial toxicity of antiglaucoma formulations: in vitro study of repeated applications. Clin Ophthalmol. 2012;6:1433-1440. doi:10.2147/OPTH.S35057

18. Organisation for Economic Co-operation and Development (OECD). Test guideline no. 492: reconstructed human cornea-like epithelium (RhCE) test method for identifying chemicals not requiring classification and labelling for eye irritation or serious eye damage. 2019. Available from: https:/www.oecd-ilibrary.org/content/publication/ 9789264242548-en. Accessed December 5, 2019.

19. Organisation for Economic Co-operation and Development (OECD). Test guideline no. 405: acute eye irritation/corrosion. 2017. Available from: https://www.oecd-ilibrary.org/content/publication/ 9789264185333-en. Accessed December 5, 2019.

20. Weil CS, Scala RA. Study of intra- and interlaboratory variability in the results of rabbit eye and skin irritation tests. Toxicol Appl Pharmacol. 1971;19(2):276-360. doi:10.1016/0041-008X(71)90112-8

21. Spielmann H, Liebsch M, S K, et al. Results of a validation study in Germany on two in vitro alternatives to the draize eye irritation test, the HET-CAM test and the 3T3 NRU cytotoxicity test. Altern Lab Anim. 1996;24:741-858.

22. Doucet O, Lanvin M, Thillou C, et al. Reconstituted human corneal epithelium: a new alternative to the Draize eye test for the assessment of the eye irritation potential of chemicals and cosmetic products. Toxicol in Vitro. 2006;20(4):499-512. doi:10.1016/j.tiv.2005.09.005

23. McNamee P, Hibatallah J, Costabel-Farkas M, et al. A tiered approach to the use of alternatives to animal testing for the safety assessment of cosmetics: eye irritation. Regul Toxicol Pharmacol. 2009;54(2):197-209. doi:10.1016/j.yrtph.2009.04.004

24. Hartung T, Bruner L, Curren R, et al. First alternative method validated by a retrospective weight-of-evidence approach to replace the Draize eye test for the identification of non-irritant substances for a defined applicability domain. ALTEX. 2010;27(1):43-51. doi:10.14573/altex.2010.1.43

25. Adriaens E, Barroso J, Eskes C, et al. Retrospective analysis of the Draize test for serious eye damage/eye irritation: importance of understanding the in vivo endpoints under UN GHS/EU CLP for the development and evaluation of in vitro test methods. Arch Toxicol. 2014;88(3):701-723. doi:10.1007/s00204-013-1156-8

26. European Parliament, Council of the European Union. Directive 2010/63/EU of the European parliament and of the council of 22 September 2010 on the protection of animals used for scientific purposes. 2010. Available from: https://eur-lex.europa.eu/legalcontent/EN/TXT/?uri=celex\%3A32010L0063. Accessed December $5,2019$.

27. Organisation for Economic Co-operation and Development (OECD). Guidance document on an integrated approach on testing and assessment (IATA) for serious eye damage and eye irritation. Series on testing \& assessment No. 263. 2017. Available from: http://www. oecd.org/officialdocuments/publicdisplaydocumentpdf/?cote $=\mathrm{ENV} /$ JM/MONO(2017)15\&doclanguage=en. Accessed August 5, 2019.

28. Alepee N, Adriaens E, Grandidier MH, et al. Multi-laboratory evaluation of SkinEthic HCE test method for testing serious eye damage/ eye irritation using solid chemicals and overall performance of the test method with regard to solid and liquid chemicals testing. Toxicol in Vitro. 2016;34:55-70. doi:10.1016/j.tiv.2016.02.014

29. Alepee N, Leblanc V, Adriaens E, et al. Multi-laboratory validation of SkinEthic HCE test method for testing serious eye damage/eye irritation using liquid chemicals. Toxicol in Vitro. 2016;31:43-53. doi:10.1016/j.tiv.2015.11.012

30. Kaluzhny Y, Kinuthia MW, Truong T, Lapointe AM, Hayden P, Klausner M. New human organotypic corneal tissue model for ophthalmic drug delivery studies. Invest Ophthalmol Vis Sci. 2018;59(7):2880-2898. doi:10.1167/iovs.18-23944

31. Epstein SP, Ahdoot M, Marcus E, Asbell PA. Comparative toxicity of preservatives on immortalized corneal and conjunctival epithelial cells. J Ocul Pharmacol Ther. 2009;25(2):113-119. doi:10.1089/jop.2008.0098 


\section{Publish your work in this journal}

Clinical Ophthalmology is an international, peer-reviewed journal covering all subspecialties within ophthalmology. Key topics include: Optometry; Visual science; Pharmacology and drug therapy in eye diseases; Basic Sciences; Primary and Secondary eye care; Patient Safety and Quality of Care Improvements. This journal is indexed on PubMed
Central and CAS, and is the official journal of The Society of Clinical Ophthalmology (SCO). The manuscript management system is completely online and includes a very quick and fair peer-review system, which is all easy to use. Visit http://www.dovepress.com/ testimonials.php to read real quotes from published authors.

Submit your manuscript here: https://www.dovepress.com/clinical-ophthalmology-journal 